\title{
Günter Johannes Stipa 1907-1993
}

Am 26. Januar 1993 ist Günter Johannes Stipa, Dozent i. R. für Finnischugrische Sprachwissenschaft an der Universität Helsinki und Honorarprofessor an der Georg-August-Universität Göttingen, im 86. Lebensjahr in Helsinki verstorben. Mit dem Tod des Gelehrten verlor die Finnougristik einen verdienstvollen Wissenschaftler, seine Fachgenossen beklagen den Verlust eines hochgeschätzten Kollegen und Freundes.

Die Fachorgane haben den Arbeiten von G. J. Stipa in Rezensionen und Diskussionsbeiträgen stets gebührende Aufmerksamkeit geschenkt. Seine Bücher, so die zuletzt erschienene große Wissenschaftsgeschichte (s.u.), werden auch zukünftig zum festen Bestandteil unserer Wissenschaft gehören. Auch Stipas beruflicher Werdegang, dessen erste Periode von theologischen und philosophischen Studien geprägt war, wurde anläßlich des 70. und 80. Geburtstages mehrfach - nicht zuletzt durch den Autor dieser Zeilen - gewürdigt. An Leben und Werk des Verstorbenen sollte auch diesmal, beim letzten Abschied, erinnert werden; es scheint mir jedoch, daß dies allein nicht genügt, daß unsere Erinnerung seiner agilen Persönlichkeit erst gerecht wird, wenn wir auch ihn selbst - nach Möglichkeit - zu Wort kommen lassen.

Und diese Möglichkeit ist da: Im Archiv der Societas Uralo-Altaica (Hamburg), die G. J. Stipa 1982 zu ihrem Ehrenmitglied wählte, werden schriftliche Zeugnisse für den vom Idealismus erfüllten Tatendrang des äußerlich unscheinbaren Mannes aufbewahrt, in dessen Redeweise stets ein Hauch von unaufdringlichem - und deshalb nie peinlichem - Pathos mitschwang. Lassen wir also ihn, zunächst durch seine Selbstbiographie, sprechen:

"Geboren am 27.11.1907 in Königshütte, Oberschlesien, und getauft auf die Vornamen Günter Johannes, absolvierte ich 1927 das 9-klassige humanistische 'Hindenburggymnasium' in Beuthen, Oberschlesien. Nach anschliessendem zweijährigem Studium im Geistlichen Seminar zu Mittelsteine Grafschaft Glatz, begann ich 1929 das Studium der Philosophie an der philos.-theol. Hochschule zu Valkenburg, Holland, und legte nach 6 Semestern 1932 die Abschlussprüfung 'De universa philosophia' ab. 1933-1940 war ich in Reval (Tallinn), Estland, (mit Unterbrechungen) als Erzieher und Lehrer tätig. Hier begann ich mit der Erlemung des Estnischen und dem Studium der finnischugrischen Sprachen, insbesondere des Ostseefinnischen (Dialektstudien an den 
'Lutser Esten', Artikel über die Livländische Chronik in 'Kiriku Elu'). Während eines 4-semestrigen Theologiestudiums in Innsbruck (1936-1938) lernte ich gleichzeitig Ungarisch und machte 1937 eine zweimonatige Studienreise nach Ungam. 1940-1941 studierte ich an der Universität Wien, wo ich die kath. Theologie absolvierte (Absolutorium)."

In dieser 'weltlichen' Selbstbiographie ging Stipa nicht näher darauf ein, $\mathrm{da} ß$ die erwähnten Studien- und Wirkungsstätten zur Ordensgemeinschaft der Societas Jesu gehörten und daß er bis 1950 Mitglied des Jesuitenordens war. Bei seiner späteren finnougristischen Forschungsarbeit kam Stipa die vielseitige, gründliche Vorbildung der Gesellschaft Jesu zugute. Ihr verdankte er eine solide Denkschulung, Klugheit im Urteil, das Bedürfnis nach vielseitiger Durchleuchtung der Probleme, Fähigkeit zur Kritik und einen gesunden Instinkt für das Plausible.

Die finnougristische Laufbahn begann G. J. Stipa relativ spät. Er hatte bereits das vierzigste Lebensjahr vollendet, als er 1949 bei Julius von Farkas und dem Slavisten Maximilian Braun in Göttingen zum Dr.phil. promovierte. Seine Dissertation "Die russischen Lehnwörter im Mokschamordwinischen und ihre Beziehungen zur russischen Sprachentwicklung" liegt im Grenzgebiet zwischen Slavistik und Finnougristik, ein Themenkreis, der ihn auch später beschäftigte.

Ende 1949 uibersiedelte Stipa nach Finnland, wo er an der Universität Helsinki ein Lektorat für Deutsch übernahm. Hier, in neuer Umgebung und neuem Wirkungskreis, hat er seine vielseitigen Fähigkeiten voll zur Entfaltung gebracht. Das Amt als Deutschlektor bedeutete für ihn eine verantwortungsvolle und zeitraubende Arbeit; trotzdem nahm er die Gelegenheit zu weiteren finnisch-ugrischen Studien wahr und betätigte sich mit zunehmendem Erfolg auf diesem Gebiet. Auf Anregung und unter der Anleitung von Professor Erkki Itkonen betrieb er permische Studien und veröffentlichte zunächst kleinere Arbeilen, Berichte und Buchbesprechungen.

In Finnland hat sich G. J. Stipa sehr rasch akklimatisiert, das Land betrachtete er nach kurzer Zeit - wie dies seine an Julius von Farkas gerichteten Briefe zeigen - als seine wahre Heimat. Kaum in Helsinki angekommen, nahm er den Briefwechsel mit seinem verehrten Göttinger Doktorvater auf, und dieser Briefwechsel riß bis zum Tode von Julius von Farkas, 1958, nicht mehr ab. Stipas im oben erwähnten Archiv aufbewahrten Briefe dokumentieren seine Aktivitäten innerhalb und außerhalb der Wissenschaft, so z. B. seine Initiative für einen finnisch-deutschen Studentenaustausch. Bereits im Brief vom 14. März 1950 berichtete er von dem Plan, wie finnische Studierende nach Göttingen und deutsche Kommilitonen nach Helsinki geschickt werden können. Der Göttinger Ordinarius griff den Plan gern auf: "Die Idee, dass finnische Studenten ihr Pflichtsemester in Göttingen absolvieren oder vorher noch zu einem Ausländerkursus eingeladen werden sollten, finde ich ganz ausgezeichnet und 
bin gem bereit, die Organisierung in die Hand zu nehmen" - antwortete er am 29. März. Die Unternehmung wurde von Erfolg gekrönt: Der im Sommersemester 1951 mit der Entsendung eines finnischen Studenten begonnene Austausch wurde in den darauffolgenden Jahren in beide Richtungen ausgedehnt und zahlenmäßig erweitert, und er hält in abgewandelter Form bis heute an.

In diesen Jahren entwickelte sich das anfängliche Lehrer-Schüler-Verhältnis zwischen ihm und Julius von Farkas zu einer vertrauensvollen Freundschaft. Stipa berichtete dem Göttinger Freund, der ihn nach Zeugnis der Briefe aus den Jahren 1957/58 zu habilitieren gedachte, ausführlich von seinem zufriedenen Leben in Finnland. Dazu gehörten auch große Wanderungen bis in den hohen Norden. Ein interessantes Dokument dieser von Naturverbundenheit inspirierten Unternehmungen ist sein am 28. Juli 1954 an Julius von Farkas geschriebener Brief, dessen einschlägiger Teil hier auszugsweise wiedergegeben wird:
"Bei strahlender Mitternachtssonne kam ich am Tenojoki an, wanderte ein we- nig in der Ailigas-Tunturi und fuhr dann - das war das Interessanteste - im Lappenboot, dem einzigen Verkehrsmittel dort, da es im Umkreise von ca. $200 \mathrm{~km}$ keine Landstrasse gibt, zunächst einen Tag lang den Tenojoki ca. $70 \mathrm{~km}$ abwärts, dabei verschiedene Lappenfamilien in ihren Fischerhütten besuchend. Telephonisch - beide Ufer entlang gehen Telephonleitungen - wurde das Mit- tagessen bei einer Lappenfamilie bestellt. Phantastisch! Reinste Naturprodukte des Landes: erstklassiger Renntierbraten, Lachs frisch aus dem Fluss (der beste der Welt) geräuchert und gebraten, hilloa (Beerenkompott) usw. ... Nach eini- gen Tagen im Lappenboot weiter bis Sirma: An den Ufern bei heller Mitter- nachtssonne fröhliche Feuer der Lachsfänger. Am Morgen weiter im Boot über die nördlichste Grenze Finnlands bei Nuorgamo hinaus bis Skipagurra. Ich wunderte mich nicht wenig, nach drei Tagen Bootsfahrt eine Brücke über den Fluss zu sehen. ... Auf dem Teno musste das flache Lappenboot streckenweise mit langen Stangen und Seilen durch heftige Strudel gesteuert werden, wobei wir Passanten uns einen Weg am Ufer bahnten. Leider beginnt man mit dem Bau einer Landstrasse am Ufer entlang, so dass nach einigen Jahren dieser viel- leicht unberührteste Teil Lapplands mit seiner regsamen Bevölkerung viel von seiner Eigenart einbüssen wird."

Infolge des Ablebens von Julius von Farkas am 12. Juli 1958 verzögerte sich Stipas Habilitation um zwei Jahre, sie erfolgte erst - unter Mitwirkung des aus Finnland auf ein Jahr nach Göttingen gekommenen Gastprofessors Aulis J. Joki - im Juni 1960. Als Habilitationsschrift legte G. J. Stipa der Philosophischen Fakultät der Göttinger Universität die Abhandlung "Funktionen der Nominalformen des Verbs in den permischen Sprachen" vor (MSFOu 121). Sie stellt die erstmalige Zusammenfassung des sehr heterogenen, weitverzweigten Materials, dessen übersichtliche Anordnung und Interpretation vom Standpunkt der finnisch-ugrischen und allgemeinen Sprachforschung dar, und sie gehört heute zu den Handbüchern unseres Faches. Die Untersuchung hat bei den Fachleuten beachtliche Anerkennung gefunden, wie dies z. B. die in den 
Ural-Altaischen Jahrbüchern erschienene Rezension des führenden Spezialisten auf diesem Gebiet, D. R. Fokos-Fuchs, zeigt (Bd. 34, 1962).

Ebenfalls mit dem permischen Kulturbereich beschäftigt sich die Studie "Der Ursprung der permischen Schrift" (ZDMG 110, 1960), die in der Fachwelt viel Aufsehen und Zustimmung erweckt hat. Mit der Studie dehnt Stipa die permisch-iranischen Berührungen auf dieses Gebiet aus, indem er aufgrund der Buchstabennamen georgische Beziehungen als Zwischenglied glaubhaft macht.

In den sechziger Jahren macht sich in Stipas Forschungsprogramm die Hinwendung zu volkskundlichen Themen bemerkbar. Im Aufsatz "Der Weltursprungsmythos des Kalevala" (Fabula 5, 1962) verfolgt er feinhörig die Motive des Weltschöpfungsmythos bis ans Mittelmeer und nach Asien, zeigt an ihrer Umgestaltung und wechselnden Kombinierung die geschichtliche Entwicklung auf und charakterisiert durch kontrastierenden Vergleich mit der Ilias Lönnrots Rolle bei der Entstehung des Kalevala und seine Arbeitsweise. Dem Aufsatz lag Stipas im Juni 1960 gehaltene Göttinger Antrittsvorlesung zugrunde. Nach Erhalt der Venia legendi für Finnisch-ugrische Philologie hielt er als Privatdozent von 1961 an in jedem Sommersemester Vorlesungen in Göttingen uber eine Reihe sprachwissenschaftlicher, volks- und kulturgeschichtlicher Themen. Ungeachtet dessen setzte er seine Lehrtätigkeit an der Universität Helsinki in vollem Umfang fort, und zwar von 1967 an zehn Jahre lang als Dozent für Finnisch-ugrische Sprachwissenschaft.

In der Forschung blieb Stipa permischen Fragen noch länger treu, besonders motiviert von seiner Forschungsreise in die Autonome Republik Komi November-Dezember 1961. In seiner Autobiographie berichtet er:

"Auf Einladung der Akademie der Wissenschaften der UdSSR und durch Vermittlung der Finnisch-Ugrischen Gesellschaft in Helsinki reiste ich zu den dem nördlichen $Z$ weig der Permier angehörenden Syrjänen nach Syktyvkar, wo ich an der dortigen Zweigstelle der Akademie handschriftliches Material studierte und von zahlreichen befragten Personen auch aus der Umgebung Sprachproben auf Magnetophonband aufnahm. Das gesammelte Sprachmaterial gedenke ich zu bearbeiten und zu veröffentlichen. Neben sprachwissenschaftlichen Untersuchungen ('Impersonalformen des Verbs') konnte ich kulturgeschichtliche Forschungsergebnisse ... zuletzt in der Untersuchung 'Zum Kulturbereich der syrjänischen Kerbkalender " publizieren".

In dieser Studie geht Stipa den Weg dieses bis nach Sibirien weit verbreiteten Zeitmessers nach und weist endlich als seinen Anfang die orthodoxen griechischen Klöster des Mittelalters auf (FUF 36, 1966/67).

In G. J. Stipas letzter Schaffensperiode - er war inzwischen außerplanmäßiger Professor (1966) bzw. Honorarprofessor (1970) der Göttinger Universität - dominieren wissenschaftsgeschichtliche Publikationen. Nach einer Reihe kleinerer Beiträge legte er 1990, gleichsam als 'Krönung' seiner erfolgreichen Bemühungen auch auf diesem Gebiet, die große zusammenfassende 
Monographie "Finnisch-ugrische Sprachforschung. Von der Renaissance bis zum Neupositivismus" (MSFOu 206) vor. "Jahrzehntelang hat Prof. Stipa für dieses Werk Material gesammelt, ist an die verschiedenen europäischen Forschungsstätten und Bibliotheken gereist und hat an dem Manuskript gearbeitet. Das eindrucksvolle Ergebnis dieser Pionierarbeit ist die erste umfassende, nicht auf ein Land beschränkte Wissenschaftsgeschichte der Finnougristik" stellte unlängst ein guter Kenner von Stipas letzten Lebensjahren fest (Klaas Ruppel in Finnisch-Ugrische Mitteilungen 16/17, 1992/93). Das letzte Buch des Verschiedenen wird zweifellos zu den grundlegenden Werken der Finnougristik gehören.

G. J. Stipas zahlreiche wissenschaftliche Publikationen, von denen hier nur ein Teil erwähnt werden konnte, zeigen eine Forscherpersönlichkeit, die durch sauberen methodischen Ansatz, das Bemühen um Motivierung sprachlicher Tatbestände durch sprachliche Mittel und das beharrliche Fragen nach dem Sinn sprachlicher Erscheinungen charakterisiert ist. Und der Mensch, der gute Kollege und der warmherzige Freund bleibt für viele von uns unvergeßlich.

ISTVÁN FUTAKY 